\title{
EDITORIAL
}

\section{CONOCER PARA TRANSFORMAR. LA INFORMACIÓN Y EL CONOCIMIENTO PARA LA EQUIDAD DE LA SALUD}

\footnotetext{
Somos conscientes de que nuestra sabiduría empieza donde la del autor termina. Y quisiéramos que nos diera respuesta, cuando todo lo que puede hacer por nosotros es excitar nuestros deseos. [...] Pero por una providencial ley singular por añadidura de la óptica de la mente (ley que significa tal vez que no podemos recibir la verdad de nadie y que debemos crearla nosotros mismos) aquello que es el término de su sabiduría no se nos presenta más que como el comienzo de la nuestra, de manera que cuando nos han dicho todo lo que podian decirnos surge en nosotros la sospecha de que todavía no nos han dicho nada.
}

Marcel Proust ${ }^{1}$

\section{Cristina Pérez Andrés}

Comité de Redacción de la Revista Española de Salud Pública. Ministerio de Sanidad y Consumo.

Con el objeto de debatir sobre el acceso al conocimiento a través de Internet para lograr una mayor equidad en el terreno de la salud, promovidos por el Centro Latinoamericano y del Caribe de Información sobre Ciencias de la Salud (BIREME/OPS/OMS) y el Centro Nacional de Información sobre Ciencias Médicas (CNICM) del Ministerio de Salud Pública de Cuba, del 23 al 27 de abril se han celebrado en la Habana la $\mathrm{Se}$ gunda Reunión de Coordinación de la Biblioteca Virtual en Salud (www.bireme.br/crics5/E/reuniao_program.htm) y el $V$ Congreso Regional de Información en Ciencias de la Salud, bajo el lema Conocer para transformar. La información y el conocimiento para la equidad de la salud (www.bireme.br/crics5/homepage.htm),

Durante la Segunda Reunión de Coordinación de la Biblioteca Virtual en Salud se elaboró la Declaración de la Habana: Hacia el Acceso Equitativo a la Información en Salud (www.bireme.br/crics5/E/declara.), cuyo texto se reproduce tras este editorial. Con la lectura de dicha declaración se clau- suró el $V$ Congreso Regional de Información en Ciencias de la Salud.

Los editores de las revistas incluidas en Scielo Salud Pública ${ }^{2}$ (www.scielosp.org) estuvimos presentes en ambas reuniones y realizamos un grupo de trabajo sobre dicha colección, con el objetivo de discutir los avances, problemas y desafíos de su implantación y su desarrollo futuro. Durante este grupo de trabajo, se perfilaron los criterios de inclusión de nuevas revistas y los editores presentes junto con los responsables de Scielo nos constituimos en Comité Consultivo para la evaluación de las revistas que soliciten su inclusión en Scielo Salud Pública.

Durante el Congreso, la exaltación de los conceptos de ciberdemocracia e inteligencia colectiva por parte del filósofo Pierre Levy, la exposición de la preocupación y de las soluciones de los responsables de Medline, de la Asociación Americana de Informática Médica y de otras instituciones, para que el ciudadano de a pié tenga una información científica que pueda en- 
tender, le han dado al congreso un tono excesivamente optimista, calificado en alguna ocasión de utópico, porque creo que es obvio que con Internet no se puede hacer nada por evitar las desigualdades sociales que no se haya podido hacer antes si hubiera habido voluntad política para ello, y que las desigualdades no se solucionan interviniendo exclusivamente en el sector de la salud, porque la falta de equidad en este ámbito es una manifestación más de la injusticia social general, aunque desde él se puedan y se deban señalar sus consecuencias para la salud de la sociedad y de los individuos.

Pensar que la brecha entre ricos y pobres no desaparecerá con Internet y que incluso puede incrementarse no creo que sea pesimista, sino más bien realista, y será conveniente contar con ello. Internet en sí misma no es más que un recurso que, como otros, hoy está en manos de las personas ricas de los países ricos, y para poder utilizar sus ventajas de cara a reducir las desigualdades sociales tendrá que estar disponible previamente allí donde sean necesarios éste y otros recursos imprescindibles para atacar la pobreza. Si en la actualidad el país de América Latina que mayor cobertura telefónica tiene es Chile, donde sólo el 25 por ciento de la población dispone de teléfono, difícilmente se puede decir que Internet vaya a solucionar las desigualdades sociales, mientras que sí se puede esperar que aumenten las diferencias entre los países con mayor acceso a la información a través de la red y los que menos lo tienen.

En cualquier caso, es necesario no olvidar, aunque no sólo en relación a Internet, que información y conocimiento no son sinónimos, y que el conocimiento es una experiencia individual que no se da necesariamente por mucha información que se tenga disponible, ya sea en forma de libros, ya sea archivada en la red.

En mi opinión, en relación con la utilización de Internet para la difusión de la infor- mación destaca muy positivamente el proyecto Scielo (www.scielo.org), que nació del deseo de aumentar la visibilidad y accesibilidad de las revistas científicas brasileñas, tanto a nivel nacional como internacional $^{3}$. Promovido por la Fundación de Protección a la Investigación del Estado de Sao Paulo (FAPESP) y el Centro Latinoamericano y de Caribe de Información en Ciencias de la salud (Bireme), posteriormente se ha extendido a otros países de América Latina (Chile y Cuba), estando prevista su implantación progresiva en la totalidad de ellos. La metodología Scielo permite la elaboración de informes bibliométricos del conjunto de revistas incluidas en la colección y con el tiempo se podrá calcular el factor de impacto de cada una de ellas. A corto plazo existirá el sitio Scielo España, que incluirá las revistas españolas de ciencias de la salud, y que se desarrollará en el marco del convenio entre BIREME/ops y la Biblioteca Nacional de Ciencias de la Salud, de la Escuela Nacional de Sanidad, del Instituto de Salud Carlos III.

Quizás en este ámbito de la salud, el de la publicación científica, Internet sí pueda disminuir desigualdades, entre ellas las que se originan en relación al hecho de que el idioma utilizado para publicar los resultados de investigaciones científicas sea mayoritariamente el inglés. Siendo las investigaciones científicas en su mayor parte financiadas con los recursos públicos de cada país, son sin embargo algunas editoriales privadas de determinados países ricos las que publican los resultados y se quedan con los derechos de autor de las publicaciones, independientemente de en qué país se hayan realizado las investigaciones. Este efecto perverso en la publicación científica se perpetúa al elaborar unos requisitos de uniformidad para revistas biomédicas ${ }^{4}$ que prohiben la publicación de los trabajos científicos en más de una revista, algo que viene a agravar el problema del idioma original en una publicación, ya que si se pudiera reproducir un artículo en diferentes revistas (como ocurre por ejemplo con las obras literarias, que son habitualmente tradu- 
cidas a diferentes idiomas para hacerlas accesibles al mayor número posible de lectores), se podría mantener la publicación en el país de origen y en su lengua, sin repercutir negativamente en el curriculum de los autores del trabajo, a causa de la valoración que, automáticamente, se hace de la calidad de un artículo dependiendo de si está publicado en inglés o no ${ }^{5}$. Esto condiciona no sólo que los investigadores de cualquier país prefieran las revistas que se publican en inglés (fundamentalmente las británicas y estadounidenses) para difundir sus trabajos, sino también que toda la bibliografía que citan en ellos sea también de artículos publicados en estas revistas. Además son estas revistas, a través del Science Citation Index, las que participan del cálculo del factor de impacto, lo que otra vez favorece que sean elegidas por los autores de cualquier país para publicar sus artículos científicos. Como consecuencia de ello, estos países tienen que pagar primero los recursos para investigar y después las revistas que tienen que comprar a Estados Unidos o Gran Bretaña si quieren conocer los resultados de las investigaciones realizadas y financiadas por ellos mismos. En este sentido, si el proyecto Scielo se institucionaliza en Latinoamérica y en España, con suficientes revistas de calidad que permitan el cálculo de un factor de impacto independiente del Science $\mathrm{Ci}$ tation Index, se conseguirá que otros idiomas diferentes del inglés, como el español y el portugués, vuelvan a ser tenidos en cuenta para la transmisión de la información científica y los investigadores de cada país podrán elegir las revistas que se editan en el suyo sin miedo a que sus trabajos dejen de tener impacto en la comunidad científica.

Tal como se ha manifestado durante las reuniones de la Habana, con relación a las publicaciones científicas en Internet, uno de los aspectos que más preocupa es quien va a controlar y cómo la calidad de lo que se publica. Recientemente hemos podido leer en la prensa ${ }^{6}$ el fraude de un investigador sudafricano que falsificó los resultados de un ensayo clínico realizado para evaluar la eficacia del tratamiento del car- cinoma de mama, utilizando altas dosis de quimioterapia junto con trasplante de médula ósea, habiendo publicado que dicho tratamiento aumentaba la supervivencia de las mujeres que padecían la enfermedad. Los falsos resultados de esta investigación fueron publicados por sus autores en una revista impresa en $1995^{7}$ y no es éste el primer caso de este tipo de fraude en una prestigiosa revista científica. Por consiguiente, hay que reconocer que en estos aspectos, como en otros, Internet reproduce los efectos perversos de las publicaciones en otros medios, pero no se le puede achacar que se originen a causa de la publicación en la red.

El acceso a la información y al conocimiento especializado por parte de los ciudadanos ha sido otro de los aspectos tratados en diferentes sesiones del congreso. Sin embargo, cuando se habla de estos ciudadanos se insiste en seguir abusando del término pacientes (pasivos) ${ }^{8}$ para referirse a los mismos, lo que resulta contradictorio con la declaración por parte de los promotores de estos programas de buscar una postura activa de los individuos en el cuidado de su propia salud, mucho más contradictorio, si cabe, cuando se habla de programas de intervención en salud pública para la prevención de enfermedades.

Por último, una de las mayores ventajas propugnadas por algunos sectores en relación con Internet es la globalización. Sería bueno tener en cuenta las palabras de Philipe Quéau, Director de la División de Información e Informática de la Unesco: ... El concepto de civilización global es un criterio parcializado, un sueño sectario de una minoría muy privilegiada (los amos globales) un pequeño subgrupo de los habitantes de este planeta [...] El principal riesgo de una civilización global es que puede terminar limitando la diversidad, al imponer poderosas normas de conducta y patrones de comportamiento. Si ello sucediera no seríamos una civilización en progreso ${ }^{9}$. 
Efectivamente, para el progreso de la humanidad existen alternativas bastante más defendibles que la globalización. Promover el mestizaje de los pueblos es una opción mucho más creativa y respetuosa con todas las culturas existentes, que al tiempo que permite su supervivencia (en lugar de reproducir sistemas autoreferentes como hace la globalización), produce el intercambio y la convivencia de experiencias sociales y culturales, a partir de las cuales no sólo se consigue que sobrevivan las culturas originales, sino que se elaboran y crean elementos nuevos que servirán al enriquecimiento de la humanidad y con él a su progreso y desarrollo basados en la pluralidad.

Internet es una herramienta inventada por los seres humanos y los resultados de su aplicación dependerán del uso que se quiera hacer de ella. La radioactividad en sí misma no es buena ni mala, pero dependiendo de los fines con los que se utilice puede servir para curar enfermedades o para matar. Con el uso de la fotografía se puede contribuir a la cultura o cometer delitos como el de la pornografía infantil. Los que falsifican datos de ensayos clínicos no han necesitado que se invente Internet para difundirlos. Las personas solidarias utilizarán Internet para seguir siéndolo. Los ricos han existido siempre y algunos van a aumentar sus riquezas gracias a Internet. La pobreza va a seguir existiendo con Internet y sin ella, porque nunca se han puesto los medios para evitarla. Por tanto Internet es una herramienta de trabajo muy útil, y en su uso se re- flejarán los valores de la humanidad pero también sus errores. Como tal puede contribuir al progreso de la civilización o a su destrucción.

\section{BIBLIOGRAFÍA}

1. Proust M. Sobre la lectura. Valencia: Pre-textos; 1997. p. 36-7.

2. C. Pérez Andrés. La Revista Española de Salud Pública en la Biblioteca Virtual Scielo. Rev Esp Salud Pública 1999; 73 (6): 665-667.

3. Packer AL, Rocha Biojone M, Antonio I, Mayumi Takemaka R, Pedroso García A, Costa da Silva A et all. Scielo: una metodología para la publicación electrónica. ACIMED 2001; 9: 9-22.

4. Comité Internacional de Directores de Revistas Médicas. Requisitos de uniformidad para manuscritos presentados a revistas biomédicas (5. ${ }^{\text {a }}$ revisión). Rev Esp Salud Pública 1997; 71 (2):89-102

5. Navarro F. El inglés, idioma internacional de la medicina. Medico Interamericano 2001; 20: 16-24.

6. Sampedro J. Cientos de españolas con cáncer de mama han sido tratadas con un método basado en un fraude. El País 2001, 1 de mayo. Sección Sociedad: 1 y 23 .

7. High-dose chemotherapy with hematopoietic rescue as primary treatment for metastatic breast cancer: a randomized trial. J Clin Oncol 1995;13:2483-9.

8. Pérez Andrés C. Humano ¿demasiado humano? Rev Esp Salud Pública 1999; 73: 1-2.

9. Quéau P. La revolución de la información: en la búsqueda del bien común. ACIMED2001; 9: 102-110. 